\title{
Anemia y deficiencia de hierro en mujeres en edad re- productiva usuarias del Hospital Regional de Villa Hayes, Paraguay
}

Jessica Riveros', Gloria Echagüe"', Santiago Evers'", Laura Mendoza"'

I. Facultad de Ciencias Químicas, Universidad Nacional de Asunción

II. Instituto de Investigaciones en Ciencias de la Salud, Universidad Nacional de Asunción

III. Hospital Regional de Villa Hayes, MSPyBS

Cómo referenciar este artículo/ How to reference this article:
Riveros J, Echagüe G, Evers S, Mendoza L. Anemia y deficiencia de hierro en mujeres en edad reproductiva usuarias del Hospital Regional de Villa Hayes, Paraguay. Mem. Inst. Investig. Cienc. Salud. $2015 ; 13(2): 26-38$

\section{RES U M E N}

La anemia es un problema de salud pública a nivel mundial. En Paraguay, existen datos limitados sobre la frecuencia de anemia en mujeres en edad reproductiva no gestantes. Este estudio piloto descriptivo de corte transverso tuvo como objetivo determinar la frecuencia de anemia y deficiencia de hierro, el estado nutricional, hábitos alimentarios y tóxicos en 99 mujeres no gestantes en edad reproductiva de 18 a 48 años que acudieron al Hospital Regional de Villa Hayes en Octubre del 2.014. La anemia se determinó tomando como punto de corte una concentración de hemoglobina $<12 \mathrm{~g} /$ dL. La deficiencia de hierro se evaluó según la saturación de la transferrina. Además, se evaluó el índice de masa corporal (IMC) y los hábitos nutricionales a través de una encuesta. La frecuencia de anemia fue de 15,1\% ( $\operatorname{IC}_{95 \%} 8,7-23,8 \%$ ), de éste el 33,3\% ( IC $_{95 \%}$ 12,8-61,6\%) correspondió a anemia ferropénica. Se observó asociación entre la presencia de anemia y los niveles disminuidos tanto del hematocrito $(<38 \%)$ como del número de glóbulos rojos $\left(<4,2 \times 10^{6} / \mathrm{uL}\right)$. Todas las mujeres con anemia ferropénica tenían peso $<64 \mathrm{~kg}$ e IMC $<24,5 \mathrm{~kg} / \mathrm{m}^{2}$. Además, se detectó una alta frecuencia de sobrepeso y obesidad $(45,4 \%)$, alto consumo de grasas, y bajo consumo de legumbres, frutas y verduras. A partir de estos resultados, se sugiere mantener la vigilancia e implementar programas que incluyan a estas mujeres, tanto para evitar deficiencias nutricionales como excesos.

Palabras clave: Anemia, deficiencia de hierro, hábitos alimentarios, IMC.

\section{Anemia and iron deficiency in reproductive age women attending the Regional Hospital of Villa Hayes, Paraguay}

\section{A B S T R A C T}

Anemia is a public health problem all around the world. In Paraguay, there are limited data on the prevalence of anemia in non-pregnant women of reproductive age. This pilot descriptive cross-sectional study had as objective to determine the frequency of anemia and iron deficiency, nutritional status, dietary and toxic habits in 99 non-pregnant women of reproductive age of 18-48 years attending the Regional Hospital of Villa Hayes in October 2014. The anemia was established on the cutoff hemoglobin concentration of $<12 \mathrm{~g} / \mathrm{dL}$. Iron deficiency was evaluated according to the transferrin saturation. Besides, the body mass index (BMI) and dietary habits were evaluated using a survey. The frequency of anemia was $15.1 \%\left(\mathrm{CI}_{95 \%} 8.7-23.8 \%\right)$, of this $33.3 \%\left(\mathrm{CI}_{95 \%} 12.8-61.6 \%\right)$ was iron-deficiency anemia. There was association between the cases of anemia and the decreased levels of hematocrit $(<38 \%)$ and red blood cell count $(<4.2106 / \mathrm{uL})$. All women with iron deficiency anemia had a weight $<64 \mathrm{~kg}$ and a BMI $<24.5 \mathrm{~kg} / \mathrm{m}^{2}$. In addi- 
tion, a high frequency of overweight and obesity (45.4\%), high consumption of fat, and low consumption legumbres, fruits and vegetables were detected. From these results, it is suggested that it is important to maintain surveillance and implement programs including these women, both to avoid nutritional deficiencies and excesses.

Keywords: Anemia, Iron deficiency, Eating habits, BMI.

\section{NTRODUCCIÓN}

La anemia representa en la actualidad uno de los problemas de Salud Pública más difundidos a nivel mundial. Entre 1.993 y 2.005, la Organización Mundial de la Salud (OMS) registró alrededor de 1,6 billones de personas con anemia en el mundo, correspondiente al $24,8 \%$ de la población (1).

La deficiencia de hierro es la causa de anemia más común en el mundo, por lo que generalmente se asume que este déficit es causante del $50 \%$ de todos los casos de anemia. La anemia a su vez puede ser causada por otras carencias nutricionales entre ellas, las de folato, vitamina B12 y vitamina A; o también debido a una inflamación aguda o crónica, a las parasitosis y las enfermedades hereditarias o adquiridas que afectan a la síntesis de hemoglobina y a la producción o la supervivencia de los eritrocitos $(1,2)$.

Factores como edad, sexo, niveles de ingesta o biodisponibilidad baja, pérdidas hemáticas, de acuerdo a la condición fisiológica, patológica y socioeconómica baja, podrían generar poblaciones más susceptibles a la deficiencia de hierro (3). Otro factor asociado al estado de las reservas de hierro es el índice de masa corporal, dependiente de los hábitos alimentarios de la población $(4,6)$.

La concentración de la hemoglobina por sí sola no puede diagnosticar la carencia de hierro, pero puede proporcionar información sobre la intensidad de la ferropenia (2); mientras que la saturación de transferrina permite evaluar mejor el estado del hierro y detectar las primeras etapas de depleción de las reservas de hierro aún cuando las concentraciones de hemoglobina continúan por encima del valor límite determinado para anemia $(3,7)$.

Hay segmentos de la población en el país con patrones de consumo de alimentos deficientes en hierro y otros micronutrientes (8); por ello, se establecieron estrategias de prevención, aunque éstas por lo general resultan muy limitadas $(9,10)$. Las mujeres en edad reproductiva no gestantes son un grupo vulnerable a este problema, ya que estas iniciativas de prevención, están más enfocadas en lactantes, niños y mujeres gestantes. Datos publicados por la OMS en el 2.006 estimaban que en Paraguay el $26,2 \%$ de este grupo de mujeres tenía anemia (1).

La población de Presidente Hayes, según el censo 2.002, es predominantemente joven. El grupo etáreo en el que se encuentra la mayor parte de ella está comprendido por menores de 30 años de edad, y un 30\% restante de la misma se encuentra dentro de los 30 a 59 años. Teniendo en cuenta sus características sociodemográficas, como bajo nivel educativo, y gran porcentaje de población indígena, es considerada una población susceptible a padecer deficiencias nutricionales (11).

En el país no hay muchos estudios que indiquen la prevalencia de anemia en la población; en especial en este grupo de mujeres, ni estudios que evalúen el origen de la anemia. Los estudios más recientes fueron llevados a cabo en el 2.012 en el Chaco donde se determinó la prevalencia en comunidades indígenas, encontrándose un 49,8\% de las mujeres y un 33,3\% de los hombres con la condición de anemia (12); y en el 2.013 en niños indígenas y no indígenas en Caazapá, siendo anémicos el $74,4 \%$ y el $45,8 \%$ de los niños, respectivamente (13).

Por todo ello, el objetivo del presente estudio fue determinar la frecuencia de anemia y deficiencia de hierro, el estado nutricional, hábitos alimentarios y tóxicos en mujeres en edad reproductiva que acuden al Hospital Regional de Villa Hayes, con el fin de 
incentivar la puesta en marcha de más campañas o programas que incluyan a este grupo y así sus necesidades puedan ser cubiertas.

\section{MATERI ALES Y MÉTODOS}

\section{Diseño del estudio}

Estudio piloto descriptivo de corte transverso realizado durante el mes de octubre del 2.014, que incluyó 99 mujeres en edad reproductiva de 18 a 48 años (OMS)(14) no gestantes que acudieron al Consultorio Clínico, de Planificación Familiar y Laboratorio, ya sea como pacientes o trabajadoras del Hospital Regional de Villa Hayes (Figura 1), dependiente del Ministerio de Salud Pública y Bienestar Social (MSP y BS). No fueron incluidas las mujeres embarazadas, amamantando o que no estén en ayunas, pacientes internados, ni con enfermedad de base (hipertensión arterial, diabetes, enfermedad celiaca) o que se hayan realizado alguna cirugía gastrointestinal, mujeres indígenas y las que no comprendieron la implicancia del estudio por alteraciones en sus facultades mentales. Antes de iniciado el estudio, el mismo fue previamente socializado con el Director del Hospital, y a través suyo con los profesionales del área de salud, quienes acompañaron todo el proceso. A todas las mujeres que cumplieron con los requisitos se les consultó si estaban de acuerdo de participar del estudio, se les explicaron los riesgos y beneficios y previamente a la toma de muestra firmaron un consentimiento informado. Luego, respondieron a una encuesta mediante una entrevista, que además incluyó la medición de talla y peso. Por último, se procedió a la extracción de muestra de sangre para el análisis de hemograma, hierro y transferrina.

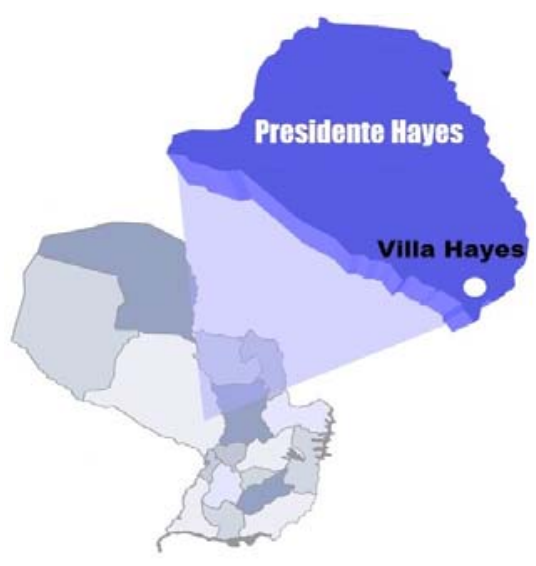

Figura 1. Ubicación del Hospital Regional de Villa Hayes en la Ciudad de Villa Hayes, Dpto. de Pdte. Hayes.

\section{Aspectos éticos}

El protocolo de investigación fue aprobado por el Comité de Ética en Investigación de la Facultad de Ciencias Químicas de la Universidad Nacional de Asunción. Toda la información se procesó en forma de códigos respetando la confidencialidad de las mujeres participantes. Los datos fueron almacenados en una computadora con acceso restringido a investigadores de este estudio.

Los resultados fueron entregados en el Hospital Regional de Villa Hayes por profesionales con experiencia en el área. Todas las mujeres con resultados fuera del rango de referencia fueron orientadas para realizar seguimiento y tratamiento en los casos necesarios.

\section{Puntos de corte establecidos}

Para establecer la frecuencia de consumo de alimentos, se determinaron límites de 
acuerdo a lo recomendado en las Guías Alimentarias del Paraguay (2.011 y 2.013) proveídas por el Instituto Nacional de Alimentación y Nutrición (INAN). Dentro de los alimentos del grupo 1 (cereales, tubérculos y derivados), se incluyeron arroz, papa, fideo y pan, los cuales se recomiendan consumir al menos una vez al día; del grupo 2, frutas, al menos 3 todos los días; grupo 3, verduras, por lo menos 2 veces al día; grupo 4, leche y yogurt, como mínimo 2 veces al día; grupo 5, carnes, 4 veces por semana; legumbres 2-3 veces por semana (18-20), pescado, 2-3 veces por semana y huevos, hasta 3 veces por semana. En cuanto al grupo 6, de azúcares, consumir hasta un equivalente de 3 cucharaditas de té al día y grupo 7, carnes grasas, en lo posible no consumirlas o hacerlo ocasionalmente (15-17). Y con respecto a los hábitos tóxicos, se preguntó a las mujeres sobre la frecuencia de consumo de alcohol y de tabaco.

El estado nutricional de las mujeres fue clasificado según indicaciones de la OMS en: bajo peso índice de masa corporal (IMC) menor a 18,50; subdividida en delgadez severa (menor a 16,00$)$ moderada $(16,00-16,99)$ y leve $(17,00-18,49)$; peso adecuado de 18,50 a 24,99; sobrepeso de 25,00 a 29,99 y obesidad mayor o igual a 30,00, subdividida a su vez en obesidad tipo I $(30,00-34,99)$, obesidad tipo II $(35,00-39,99)$, obesidad tipo III (mayor a 40) medidos en $\mathrm{Kg} / \mathrm{m}^{2}$ (18).

El volumen de sangre venosa extraída para las determinaciones fue de $5 \mathrm{~mL}$ ( $2 \mathrm{~mL}$ de sangre para el hemograma, y $3 \mathrm{~mL}$ para obtener suero). Los biomarcadores que fueron analizados a partir de una muestra de sangre fueron: hemoglobina $(\mathrm{Hb})$, hematocrito (Hto), glóbulos rojos, volumen corpuscular medio (VCM), hemoglobina corpuscular media (HCM), hierro sérico y capacidad total de fijación del hierro (CTFH Transferrina), y saturación de la transferrina.

Los parámetros hematológicos fueron cuantificados por el método automatizado realizado en el contador hematológico Mindray BC-3000Plus (Shenzhen-China) a partir de sangre total anticoagulada, y analizados en el Hospital Regional de Villa Hayes.

La determinación de hierro sérico y CTFH se realizó a partir de suero, siguiendo las instrucciones detalladas en los insertos de los reactivos de Wiener (Rosario-Argentina) por el método cuantitativo colorimétrico realizado en el autoanalizador Wiener CB350i del Instituto de Investigaciones en Ciencias de la Salud, Universidad Nacional de Asunción.

La saturación de la transferrina se determinó a partir de los valores de hierro sérico y $\mathrm{CTFH}$, puesto que, como su descenso es más pronunciado de lo que cabe esperar del descenso de hierro sérico, es un mejor indicador de la deficiencia de hierro (7). Los valores utilizados como punto de corte a partir de los cuales fueron considerados normales para el rango etario de las mujeres incluidas en el estudio fueron hemoglobina $\geq 12,0 \mathrm{~g} / \mathrm{dL}(2,19)$, hematocrito $\geq 38 \%$, glóbulos rojos $\geq 4,2 \times 10^{6} / \mathrm{uL}$ (19), volumen corpuscular medio $(\mathrm{VCM}) \geq 80 \mathrm{fL},(\mathrm{HCM}) \geq 27 \mathrm{pg} /$ célula $(20)$, hierro sérico $\geq 50 \mu \mathrm{g} / \mathrm{dL}$, $\mathrm{CTFH} \leq 400 \mu \mathrm{g} / \mathrm{dL}$, saturación de la transferrina $\geq 20 \%$ (los puntos de corte utilizados como normales para la concentración de hierro sérico, CTFH y saturación de la transferrina fueron establecidos con base en los limites de los rangos de referencia definidos por el fabricante).

Para clasificar la anemia según su gravedad se clasificó como leve: $11,0-11,9 \mathrm{~g} / \mathrm{dL}$, moderada: 8,0-10,9 g/dL, grave: menos de $8,0 \mathrm{~g} / \mathrm{dL}(2)$.

Se clasificó como anemia ferropénica cuando los valores tanto de hemoglobina como de índice de saturación estuvieron por debajo del punto de corte establecidos para cada parámetro, y como anemia no ferropénica cuando la concentración de hemoglobina estaba por debajo y el índice de saturación era igual o mayor al valor de corte a partir del cual fue considerado común. Una deficiencia de hierro se estableció cuando se obtuvo un índice de saturación por debajo y hemoglobina igual o mayor al punto de corte. 


\section{Análisis estadístico}

El análisis de los datos se realizó empleando procedimientos de estadística descriptiva y analítica utilizando el programa Epi Info ${ }^{\mathrm{TM}} 7$ (CDC, Atlanta).

Para determinar asociaciones entre las características socio-demográficas, económicas, antropométricas, y hematológicas con la presencia de anemia, así como con deficiencia de hierro, fue utilizado el test de Chi cuadrado, considerándose estadísticamente significativa los valores de $\mathrm{p} \leq 0,05$. Las variables cuantitativas (edad, ingreso per cápita, años de estudio, peso, talla, IMC, hematocito, glóbulos rojos, VCM, HCM) fueron convertidas a variables dicotómicas a fin de realizar el análisis por el test de chi cuadrado. El punto de corte fue seleccionado con base en los valores de las medianas observadas en la población de mujeres (edad, ingreso per cápita, años de estudio, peso, talla, IMC), y con base en puntos de corte establecidos como normales (en el caso de las variables hematológicas). Las medianas fueron expresadas con un $\mathrm{IC}_{25 \%-75 \% \text { y las }}$ medias con un $\mathrm{IC}_{95 \%}$.

\section{RESULTADOS}

En relación a las características sociodemográficas, las 99 mujeres incluidas presentaron un rango de edad de 18 a 48 años, con una mediana de 28 años ( IC $_{25 \%-75 \%}$ 23-35 años). Se observó una mayor frecuencia (46,5\%) de mujeres entre los 20 y 29 años. Se evidenció una mediana de 10 años de estudio y una mayor frecuencia procedente de zona urbana. La mediana del ingreso mensual per cápita fue de 428.571 Gs (IC $25 \%-75 \%$ 250.000-750.000 Gs). Cabe resaltar que el $97 \%$ de ellas manifestó que accede a los servicios del hospital al menos una vez al año, y se observó que el 30\% vive en pobreza extrema. Estos datos se muestran en la Tabla 1.

Según el IMC el 7,1\% presentaba bajo peso, el $29,3 \%$ y el $16,1 \%$ tenían sobrepeso y obesidad, respectivamente (Tabla 2). La mediana de los parámetros de hemoglobina, recuento de glóbulos rojos, hematocrito, VCM, HCM, hierro sérico, transferrina (CTFH) y saturación de la transferrina se presenta en la Tabla 2.

En 11,1\% (IC $95 \% 5,7-19,0)$ de las mujeres $(11 / 99)$ se observó deficiencia de hierro sin anemia y $15,1 \%$ (IC $95 \% 8,7-23,8 \%$ ) de mujeres (15/99) presentó anemia, de las cuales el $80 \%$ (IC $95 \% 51,9-95,7 \% \%)$, o $12 / 15$ mujeres presentó anemia leve. Además, se detectó anemia ferropénica en 5/15 mujeres (33,3\% IC $95 \% 12,8-61,6 \%$ ). (Tablas 2 y 3 ).

Todas las mujeres con anemia ferropénica presentaron un peso por debajo de $64 \mathrm{~kg}$ $(p=0,02)$ y un IMC $\leq 24,5 \mathrm{~kg} / \mathrm{m}^{2}(p=0,03)$. Se observó que gran parte de este mismo grupo de mujeres (con anemia ferropénica) también presentó un hematocrito menor a $38 \%(p<0,05)$ y recuento de glóbulos rojos por debajo de $4,2 \times 10^{6} / \mathrm{uL}(p<0,05)$, esto al igual que las mujeres que tenían anemia no ferropénica con un hematocrito menor a $38 \%(p<0,05)$ y número de glóbulos rojos por debajo de $4,2 \times 10^{6} / \mathrm{uL}(\mathrm{p}<0,05)$ (Tabla 4$)$.

Los alimentos más consumidos fueron pan $(86,9 \%)$, huevo $(79,8 \%)$ y azúcar $(75,8 \%)$; y entre los que tuvieron menor aceptación estuvieron arroz $(9,1 \%)$, fideo $(9,1 \%)$, frutas $(15,2 \%)$, verduras $(31,3 \%)$, carne magra $(23,2 \%)$, legumbres $(24,2 \%)$, y pescados $(38,4 \%)$, puesto que la mayoría de las mujeres consume estos alimentos por defecto. Un elevado porcentaje de mujeres consume carne grasa por exceso (57,6\%). Estos resultados se muestran en forma independiente en la Tabla 5 y agrupados en la Figura 2.

En cuanto a los hábitos tóxicos se observó que $56,6 \%$ (56/99) consumía bebidas alcohólicas, de éste $67,9 \%$ (38/56) consumía de 1 a 3 veces al mes, mientras que las restantes lo hacían más de 4 veces. Con respecto a la frecuencia de fumadoras fue de $5,0 \%$ (5/99), de éste el 40\% (2/5) manifestó hacerlo todos los días, y las restantes solo ocasionalmente. 
Tabla 1. Características sociodemográficas y económicas de mujeres en edad reproductiva no gestantes ( $n=99$ mujeres).

\begin{tabular}{|c|c|c|}
\hline Características & $\mathbf{n}$ & $\%$ \\
\hline Edad (Años) (mediana; rango intercuartil) & 28 & $23-35$ \\
\hline$<20$ & 10 & 10,1 \\
\hline $20-29$ & 46 & 46,5 \\
\hline $30-39$ & 29 & 29,3 \\
\hline$\geq 40$ & 14 & 14,1 \\
\hline \multicolumn{3}{|l|}{ Estado civil } \\
\hline Casada o en pareja & 68 & 68,7 \\
\hline Divorciada o separada & 2 & 2,0 \\
\hline Soltera & 29 & 29,3 \\
\hline Escolaridad en años (mediana; rango intercuartil) & 10 & $6-13$ \\
\hline Primaria & 32 & 32,3 \\
\hline Secundaria & 41 & 41,4 \\
\hline Terciaria & 26 & 26,3 \\
\hline \multicolumn{3}{|l|}{ Ocupación } \\
\hline Con empleo remunerado & 34 & 34,3 \\
\hline Desempleado & 65 & 65,7 \\
\hline $\begin{array}{l}\text { Ingreso mensual per cápita en guaraníes (mediana; } \\
\text { rango intercuartil) }\end{array}$ & 428.571 & $250.000-750.000$ \\
\hline Pobre extremo ${ }^{a}$ & 30 & 30,3 \\
\hline Pobre no extremo ${ }^{\mathrm{b}}$ & 18 & 18,2 \\
\hline No pobre & 51 & 51,5 \\
\hline \multicolumn{3}{|l|}{ Ubicación de la vivienda } \\
\hline Zona Urbana & 74 & 74,8 \\
\hline Zona Rural & 25 & 25,2 \\
\hline \multicolumn{3}{|l|}{ Hacinamiento $^{d}$} \\
\hline $\mathrm{Si}$ & 17 & 17,2 \\
\hline No & 82 & 82,8 \\
\hline \multicolumn{3}{|l|}{ Acceso a los servicios del Hospital } \\
\hline Primera vez & 3 & 3,0 \\
\hline Semanalmente & 9 & 9,1 \\
\hline Mensualmente & 51 & 51,5 \\
\hline Anualmente & 36 & 36,4 \\
\hline
\end{tabular}

a,b,c Encuesta Permanente de Hogares 2013, establece dos puntos de corte Línea de Pobreza Total (LPT) y Línea de Pobreza Extrema (LPE); clasificado con base en estos puntos de corte en pobre extremo (Ingreso per cápita menor que LPT y LPE), pobre no extremo (ingreso per cápita menor que LPT pero mayor que LPE) y no pobre (ingreso per cápita mayor que LPT y LPE). Valores de LPE y LPT según la zona donde reside: Urbano $\mathrm{LPE}=279.524$ Gs y $\mathrm{LPT}=435.067 \mathrm{Gs}$, y Rural $\mathrm{LPE}=258.654 \mathrm{Gs}$ y $\mathrm{LPT}=375.801 \mathrm{Gs}$ (33)

${ }^{d}$ El hacinamiento fue considerado como un indicador de pobreza (indicador de insatisfacción), y establecido cuando en una habitación dormían más de 3 personas (34). 
Tabla 2. Análisis de parámetros nutricionales de mujeres en edad reproductiva no gestantes. $n=99$

\begin{tabular}{|c|c|c|c|}
\hline Parámetros nutricionales & Mediana & Rango intercuartil & n (\%) \\
\hline Peso (en Kg) & 64 & $54-72$ & \\
\hline Talla $\left(\right.$ en $\mathbf{m}^{2}$ ) & 1,60 & $1,56-1,63$ & \\
\hline I MC $\left(\mathbf{K g} / \mathbf{m}^{2}\right)^{\mathrm{a}}$ & 24,5 & $20,9-27,9$ & \\
\hline Bajo peso & & & $7(7,1)$ \\
\hline Delgadez severa & & & 0 \\
\hline Delgadez moderada & & & $3(3,0)$ \\
\hline Delgadez leve & & & $4(4,1)$ \\
\hline Rango normal & & & $47(47,5)$ \\
\hline Sobrepeso & & & $29(29,3)$ \\
\hline Obesidad & & & $16(16,1)$ \\
\hline Obesidad clase I & & & $9(9,1)$ \\
\hline Obesidad clase II & & & $4(4,0)$ \\
\hline Obesidad clase III & & & $3(3,0)$ \\
\hline Hemoglobina ( g/ dL) & 12,9 & $12,3-13,5$ & \\
\hline Sin anemia & & & $84(84,9)$ \\
\hline Anemia leve & & & $12(12,1)$ \\
\hline Anemia moderada & & & $2(2,0)$ \\
\hline Anemia grave & & & $1(1,0)$ \\
\hline Hematocrito (\% ) & 39,5 & $37,9-40,9$ & \\
\hline Glóbulos rojos (x10\%/uL) & 4,49 & $4,27-4,71$ & \\
\hline $\operatorname{VCM}(\mathbf{f L})^{\mathrm{b}}$ & 88,1 & $85,8-90,8$ & \\
\hline $\operatorname{HCM}(p g)^{c}$ & 28,8 & $27,8-29,7$ & \\
\hline Hierro sérico ( $u L / d L)$ & 93,4 & $75,0-109,0$ & \\
\hline $\begin{array}{l}\text { Capacidad total de fijación de hierro - } \\
\text { transferrina ( } u L / d L)\end{array}$ & 312,0 & $282,0-350$ & \\
\hline Saturación de la transferrina (\%) & 29,2 & $24,5-35,1$ & \\
\hline
\end{tabular}

ándice de masa corporal ${ }^{b}$ Volumen corpuscular medio ${ }^{\mathrm{C}} \mathrm{Hemoglobina}$ corpuscular media.

Tabla 3. Frecuencia de mujeres en edad reproductiva con deficiencia de hierro (ferropenia) según condición de anemia. $n=99$

\begin{tabular}{llll}
\hline Frecuencia & $\mathbf{n}$ & $\mathbf{\%}$ & $\mathbf{I ~}_{\mathbf{9 5 \%}}$ \\
\hline Anemia ferropénica & 5 & 31,2 & $1,7-11,4$ \\
Anemia no ferropénica & 10 & 12,0 & $5,0-17,8$ \\
Deficiencia de hierro sin anemia & 11 & 68,8 & $5,7-19,0$ \\
Sin anemia- sin deficiencia de hierro & 73 & 73,4 & $63,9-82,1$ \\
\hline
\end{tabular}


Tabla 4. Características sociodemográficas, económicas, antropométricas y hematológicas según la condición de ferropenia y anemia

\begin{tabular}{|c|c|c|c|c|c|c|c|}
\hline Características & $\mathbf{n}$ & $\begin{array}{l}\text { Anemia } \\
\text { ferropéni- } \\
\text { ca }\end{array}$ & $\begin{array}{l}\text { Valor } \\
\mathbf{p}\end{array}$ & $\begin{array}{l}\text { Anemia no } \\
\text { ferropénica }\end{array}$ & $\begin{array}{l}\text { Valor } \\
\text { p }\end{array}$ & $\begin{array}{l}\text { Deficiencia de } \\
\text { hierro sin ane- } \\
\text { mia }\end{array}$ & $\begin{array}{l}\text { Valor } \\
\mathbf{p}\end{array}$ \\
\hline Edad (años) & & & 0,68 & & 0,71 & & 0,97 \\
\hline$<28$ & 49 & $3(6,1)$ & & $6(12,2)$ & & $6(12,2)$ & \\
\hline$\geq 28$ & 50 & $2(4,0)$ & & $4(8,0)$ & & $5(10,0)$ & \\
\hline $\begin{array}{l}\text { Ingreso per cápita(gs/ } \\
\text { mes) }\end{array}$ & & & 1,00 & & 0,09 & & 0,54 \\
\hline$<428.571$ & 49 & $2(4,0)$ & & $2(4,1)$ & & $4(8,2)$ & \\
\hline$\geq 428.571$ & 50 & $3(6,0)$ & & $8(16,0)$ & & $7(14,0)$ & \\
\hline Años de estudio & & & 0,37 & & 0,10 & & 0,70 \\
\hline$<10$ & 46 & $1(2,2)$ & & $2(4,3)$ & & $4(8,7)$ & \\
\hline$\geq 10$ & 53 & $4(7,5)$ & & $8(15,1)$ & & $7(13,2)$ & \\
\hline Ubicación de la vivienda & & & 1,00 & & 0,45 & & 0,84 \\
\hline Zona Urbana & 74 & $4(5,4)$ & & $6(8,1)$ & & $8(10,8)$ & \\
\hline Zona Rural & 25 & $1(4,0)$ & & $4(16,0)$ & & $3(12,0)$ & \\
\hline Hacinamiento & & & 0,66 & & 1,00 & & 0,17 \\
\hline $\mathrm{Si}$ & 17 & $1(5,9)$ & & $1(5,9)$ & & $4(23,5)$ & \\
\hline No & 82 & $4(4,9)$ & & $9(11,0)$ & & $7(8,5)$ & \\
\hline Peso (Kg) & & & 0,02 & & 0,98 & & 0,34 \\
\hline$<64$ & 45 & $5(11,1)$ & & $4(8,9)$ & & $3(6,7)$ & \\
\hline$\geq 64$ & 54 & 0 & & $6(11,1)$ & & $8(14,8)$ & \\
\hline Talla (m) & & & 1,00 & & 0,62 & & 0,86 \\
\hline$<1,60$ & 47 & $2(4,3)$ & & $6(12,8)$ & & $5(10,6)$ & \\
\hline$\geq 1,60$ & 52 & $3(5,8)$ & & $4(7,7)$ & & $6(11,5)$ & \\
\hline I MC ( $\left.\mathrm{Kg} / \mathbf{m}^{2}\right)$ & & & 0,03 & & 0,76 & & 0,97 \\
\hline$<24,5$ & 48 & $5(10,4)$ & & $4(8,3)$ & & $5(10,4)$ & \\
\hline$\geq 24,5$ & 51 & 0 & & $6(11,8)$ & & $6(11,8)$ & \\
\hline Hematocrito (\%) & & & $<0,05$ & & $<0,05$ & & 0,06 \\
\hline$<38$ & 25 & $5(20,0)$ & & $10(40,0)$ & & 0 & \\
\hline$\geq 38$ & 74 & 0 & & 0 & & $11(14,9)$ & \\
\hline Glóbulos rojos ( $\left.10^{6} / \mathrm{uL}\right)$ & & & $<0,05$ & & $<0,05$ & & 0,12 \\
\hline$<4,2$ & 19 & $3(15,8)$ & & $7(36,8)$ & & 0 & \\
\hline$\geq 4,2$ & 80 & $2(2,5)$ & & $3(3,8)$ & & $11(13,8)$ & \\
\hline VCM (fL) & & & 0,10 & & 0,19 & & 1,00 \\
\hline$<80$ & 2 & $1(50,0)$ & & $1(50,0)$ & & 0 & \\
\hline$\geq 80$ & 97 & $4(4,1)$ & & $9(9,3)$ & & $11(11,3)$ & \\
\hline HCM (pg) & & & 0,08 & & 1,00 & & 1,00 \\
\hline$<27$ & 10 & $2(20,0)$ & & $1(10,0)$ & & $1(10,0)$ & \\
\hline$\geq 27$ & 89 & $3(3,4)$ & & $9(10,1)$ & & $10(11,2)$ & \\
\hline
\end{tabular}


Tabla 5. Consumo de alimentos según guías alimentarias del país. $n=99$

\begin{tabular}{llllll}
\hline $\begin{array}{l}\text { Tipo de alimen- } \\
\text { to* }\end{array}$ & $\begin{array}{l}\text { Consumo sema- } \\
\text { nal } \\
\text { mediana, rango } \\
\text { intercuartil }\end{array}$ & $\begin{array}{l}\text { Consumo } \\
\text { adecuado } \\
\mathbf{n}(\%)\end{array}$ & $\begin{array}{l}\text { Consume } \\
\text { por defecto } \\
\mathbf{n}(\%)\end{array}$ & $\begin{array}{l}\text { Consume } \\
\text { por exce- } \\
\text { so } \\
\mathbf{n}(\%)\end{array}$ & $\begin{array}{l}\text { No } \\
\text { consume } \\
\mathbf{n}(\%)\end{array}$ \\
\hline -Arroz & $3(2-4)$ & $9(9,1)$ & $89(89,9)$ & -- & $1(1,0)$ \\
-Papa & $5(2-7)$ & $48(48,5)$ & $49(49,5)$ & -- & $2(2,0)$ \\
-Fideo & $3(2-3)$ & $9(9,1)$ & $86(86,9)$ & -- & $4(4,0)$ \\
-Pan & $14(7-21)$ & $86(86,9)$ & $6(6,0)$ & -- & $7(7,1)$ \\
-Frutas y Jugos & $7(3-14)$ & $15(15,2)$ & $82(82,8)$ & -- & $2(2,0)$ \\
-Verduras & $7(7-14)$ & $31(31,3)$ & $68(68,7)$ & -- & 0 \\
-Leche o Yogurt & $7(7-14)$ & $48(48,5)$ & $43(43,4)$ & -- & $8(8,1)$ \\
-Carne Magra & $2(2-5)$ & $23(23,2)$ & $47(47,5)$ & $19(19,2)$ & $10(10,1)$ \\
-Legumbres secas & $1(0-2)$ & $24(24,2)$ & $55(55,6)$ & $6(6,1)$ & $14(14,1)$ \\
-Huevos & $3(3-3)$ & $79(79,8)$ & -- & $20(20,2)$ & 0 \\
-Pescado & $3(0-3)$ & $38(38,4)$ & 0 & -- & $61(61,6)$ \\
-Azúcar & $28(28-28)$ & $75(75,8)$ & 0 & $24(24,2)$ & 0 \\
-Carne Grasa & $3(1-3)$ & $42(42,4)$ & -- & $57(57,6)$ & 0 \\
\hline
\end{tabular}

*Las frecuencias fueron realizadas teniendo en cuenta cada alimento de forma individual según las recomendaciones de las guías alimentarias.

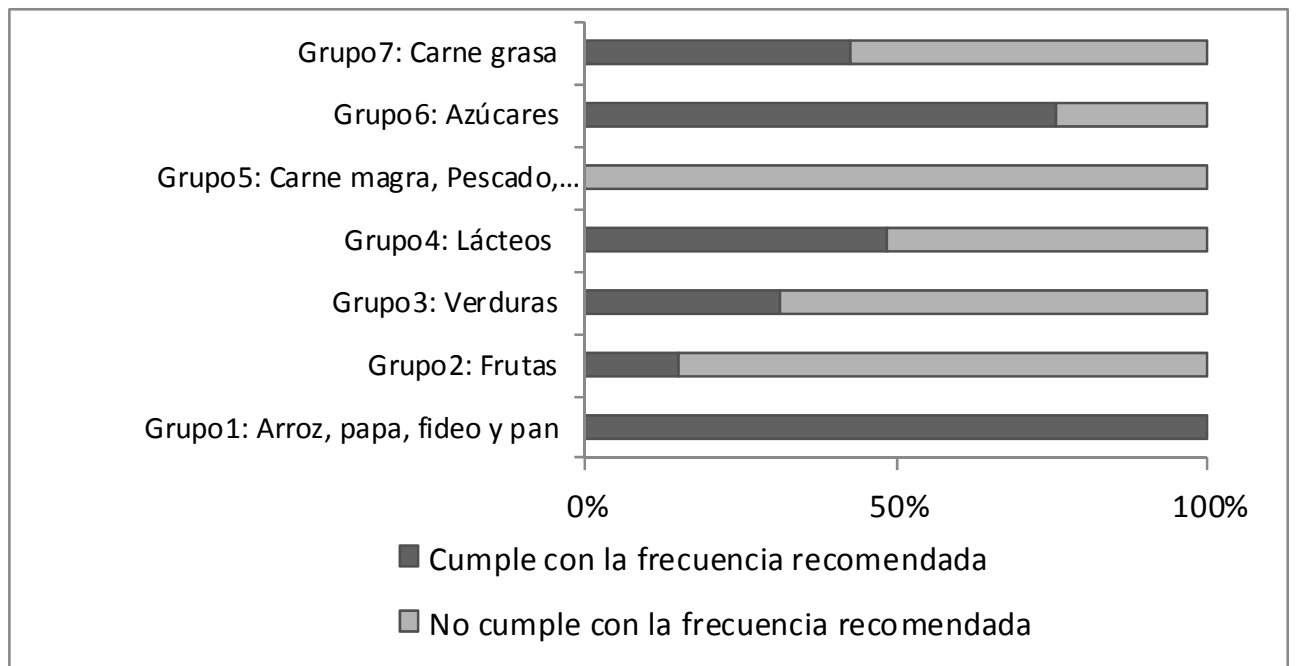

Figura 2. Frecuencia de consumo por grupo de alimentos

\section{DISCUSIÓN}

En Paraguay, al igual que en la mayoría de los países de Latinoamérica, las intervenciones nutricionales para prevenir o tratar la anemia están dirigidas especialmente a niños y mujeres gestantes (9). No existen programas que centren atención sobre las mujeres en edad fértil. Esto hace a estas últimas más susceptibles a padecer dicha enfermedad durante el embarazo. Por ello, el presente estudio analizó la frecuencia de anemia y deficiencia de hierro en este grupo.

La frecuencia de anemia $(15,1 \%)$ observada en el presente estudio se encuentra por 
debajo de las estimadas por la OMS para el grupo de mujeres en edad reproductiva no gestante, cuya estimación es del 30,2\%. De igual manera, se encuentra por debajo de la cifra publicada en el 2.006 por la OMS para el Paraguay en estas mujeres, que fue de $26,2 \%$ (1). Sin embargo, la frecuencia de anemia encontrada en la población estudiada constituye aún un problema de Salud Pública, dado que la OMS establece como cifra a considerar de importancia a partir del $5 \%$ de la población con la condición (2), por lo que se debe establecer una vigilancia continua.

Resultado similar se observó en un estudio realizado en México donde la prevalencia de anemia fue disminuyendo a través de los años, progresivamente hasta llegar al $15 \%$ (21), y en otro llevado a cabo en Chile, donde en la última década se registró solo un $10 \%$ de casos (22).

La mayoría de las mujeres en el presente estudio presentaron índices hematimétricos normales. Aunque este estudio se realizó a partir de mujeres que acuden a un hospital y puede presentar sesgos, los datos obtenidos se encuentran en concordancia con un estudio realizado por Echagüe et al. que en el 2.003 observaron que mujeres aparentemente sanas que acuden a los bancos de sangre en Asunción presentaban índices hematimétricos dentro del rango considerado normal (23).

Dentro de la frecuencia de anemia encontrada en la población de mujeres estudiada, el $5 \%$ de ellas presentó anemia ferropénica, correspondiendo al 33,3\% de los casos de anemia encontrados; similar porcentaje se obtuvo en un estudio realizado en Colombia, donde del total de casos de anemia observados, el 32,8\% correspondió a la ferropénica (24), pero fue inferior al $50 \%$ estimado por la OMS (1). Estas diferencias se podrían deber a la diferencia entre las características de las poblaciones analizadas relacionadas con los hábitos alimentarios (4).

En cuanto a la deficiencia de hierro, la frecuencia fue de $11,1 \%$ en el estudio, cercana al resultado obtenido en el mismo estudio realizado en Chile mencionado anteriormente, donde el $10 \%$ de las mujeres tenían deficiencia de hierro (22).

Al establecer asociaciones entre anemia ferropénica y las características antropométricas cabe resaltar que se encontró que las mujeres que tenían un índice de masa corporal dentro del rango normal y/o por debajo de $24,5 \mathrm{~kg} / \mathrm{m} 2$ presentaban con mayor frecuencia anemia ferropénica. Resultados similares se obtuvieron en un estudio llevado a cabo en adolescentes (5). Esto puede deberse a que niveles bajos de hierro ocasionan una disminución en apetito y por causa una disminución en el peso (4).

Sin embargo, esta relación no se verificó entre los casos de deficiencia de hierro, y características antropométricas, debido a que posiblemente estos casos de deficiencia de hierro podrían estar en sus etapas iniciales de depleción.

Cabe resaltar que se observó una baja frecuencia de mujeres con bajo peso, por el contrario, el $45,4 \%$ de las mujeres presentó sobrepeso u obesidad. Según datos de la OMS del 2.008, en el país había un $20,8 \%$ de mujeres obesas (25). En los últimos años, esta tendencia creciente hacia la obesidad ya se ha observado en toda Latinoamérica, donde el Paraguay posee uno de los porcentajes más altos de sobrepeso y obesidad en las mujeres de más de 15 años (26).

La medición de la frecuencia de consumo de alimentos otorga un panorama de los alimentos de mayor aceptación dentro de este grupo de mujeres, resaltando entre los de mayor consumo el pan. Las frutas, verduras, legumbres, pescados y carne magra tienen baja aceptación, ya que un porcentaje bajo de ellas come según lo recomendado, y las restantes las consume por debajo de ello, y son estos últimos los alimentos que más aporte de hierro brindan (27). Por este perfil tendiendo a deficiente se esperaba una mayor frecuencia de anemia ferropénica pero el bajo porcentaje observado en este estudio puede deberse al resultado del cumplimiento de la legislación vigente (según decreto 20830/1998) en el país que obliga a la fortificación de la harina de trigo comercializada con hierro y vitaminas (10), siendo este componente principal de los panificados, los cuales tuvieron gran aceptación dentro de este grupo. Al mismo tiempo 
cabe mencionar que Chile ha tenido un descenso en el número de casos de anemia ferropénica luego que la misma normativa de fortificación se pusiera en marcha (22).

En contrapartida es preocupante el alto consumo de carnes grasas y a su vez la alta frecuencia de sobrepeso y obesidad, haciendo frente al alto porcentaje de mujeres que viven en situación de pobreza (48,5\%). En la actualidad, las mujeres integran el grupo más afectado por la obesidad, problema que puede deberse a la desigualdad social, donde intervienen factores sociales, y biológicos, en conjunto con la desventaja de pertenecer en muchos casos a un nivel socio-económico bajo. La condición de pobreza por lo general hace que ellas seleccionen alimentos pobres en fibras y ricos en carbohidratos complejos, azúcares y grasas (en especial ácidos grasos trans, saturados y colesterol) que le permiten satisfacer su apetito y no así llevar una nutrición adecuada, esto debido a que los precios elevados de las frutas, vegetales frescos y otros alimentos de alta calidad nutricional, les son inaccesibles (28).

Aunque ninguna de las mujeres con sobrepeso u obesidad participantes de este estudio presentó deficiencia de hierro o anemia ferropénica, ellas no están exentas de presentar deficiencias nutricionales. Un estudio realizado en niños y adolescentes determinó que los niños y/o adolescentes con mayor índice de masa corporal, presentaban las reservas de hierro deplecionadas, debido a la inactividad física y dieta inadecuada con alimentos de bajo contenido de hierro que tenían (6). Además, la obesidad se asocia a otras complicaciones como síndrome metabólico, desregulaciones hormonales, alteraciones en la hemostasias, entre otros, que pueden causar complicaciones a la hora del parto, incluso promover la aparición de diabetes gestacional, preclampsia, macrosomía y trauma fetal $(29,30)$. Por tanto, la alta frecuencia de sobrepeso y obesidad observada en el presente estudio sugiere que es preciso realizar la promoción de consumo de alimentos ricos en minerales y vitaminas y bajo consumo de alimentos grasos, mediante la educación nutricional dirigida a la población a fin de fortalecer el sistema de salud y evitar complicaciones futuras por este mal.

A pesar de que el presente es un estudio hospitalario existen ciertas características analizadas que concuerdan con lo observado en el censo 2.002, donde la población de Presidente Hayes era predominantemente joven, siendo mayoritario el grupo etario menor de 30 años de edad, similar con lo hallado en este estudio (56,6\%) (11). Al mismo tiempo se encuentra en concordancia con los datos observados en la encuesta permanente de hogares en cuanto al nivel de educación, donde ha disminuido el número de analfabetos hasta una cifra de $10,1 \%$ en mujeres de 15 o más años. Por otro lado, el ingreso mensual per cápita encontrado es superior a los registrados por el Censo Nacional, debido a que este estudio solo incluyó a mujeres no indígenas (31).

Es importante mencionar que en el 2.007 el acceso a los servicios de salud se ha incrementado, llegando en el 2.011 a un promedio de consultas de 2,5 veces por año por persona según el Ministerio de Salud Pública, viéndose similar respuesta entre las mujeres encuestadas, siendo que el $97,0 \%$ de ellas consultaba en dichos servicios al menos una vez al año. Esto podría deberse al ofrecimiento de servicios de salud gratuitos en el hospital (32).

En conclusión la frecuencia de anemia del $15,1 \%$ es considerada como magnitud de importancia en la población analizada. Estos resultados sugieren que son necesarias la vigilancia continua y la implantación de nuevas estrategias de prevención de la anemia ferropénica y no ferropénica, así como la deficiencia de hierro, que incluyan a este grupo de mujeres, a fin de detectar tempranamente la anemia en ellas y así evitar las complicaciones asociadas a este cuadro. Por otro lado, la alta frecuencia de sobrepeso y obesidad observada sugiere la necesidad de fortalecer programas para mejorar el aporte de nutrientes en mujeres en edad reproductiva favoreciendo a la generación de reservas corporales adecuadas, aumentando la capacidad para responder a las altas demandas del embarazo; fomentando además el llevar una dieta adecuada, sin deficiencias ni excesos. 
Agradecimientos. A las Dras. Raquel Aguilar, Celia Olmedo, Mónica Silguero, Karen Quiñónez, Elena Insfrán, y Carolina Ruíz; a la Licenciada Martha Marecos y a las Técnicas de Laboratorio Amanda Núñez y Nahir Núñez quienes acompañaron la fase de reclutamiento y de los análisis clínicos llevados a cabo en el Hospital Regional de Villa Hayes.

A la Bioq. Clínica Lourdes Rivas y el Dr. Jorge Zenteno, quienes acompañaron los análisis clínicos realizados en el Instituto de Investigaciones en Ciencias de la Salud (IICS-UNA).

\section{REFERENCI AS BI BLI OGRAFICAS}

1. Benoist $B$ de, McLean E, Egli I, Cogswell M, editors. Worldwide prevalence of anaemia 1993-2005/Internet/. Ginebra: WHO, 2008. [citado 29 Ago 2014]. Disponible en: http:// w h q I i b d o c. w h o. i n t / publications/2008/9789241596657_eng.pdf

2. Organización Mundial de la salud (OMS). Concentraciones de hemoglobina para diagnosticar la anemia y evaluar su gravedad / Internet/. Ginebra: OMS; 2011. [citado 29 Ago 2014]. Disponible en: http:// www. who.int/vmnis/indicators/ haemoglobin_es.pdf

3. United Nations Children's Fund, United Nations University, Workd Health Organization. Iron deficiency anemia, assessment, prevention and control: A guide for programme managers /Internet/. Geneva: WHO; 2001. [citado 10 Sep 2014]. Disponible en: http:// apps.who.int/iris/bitstream/10665/66914/1/ WHO_NHD_01.3.pdf?ua =1

4. Chaparro CM. La anemia entre adolescentes y mujeres adultas jóvenes en América Latina y El Caribe: Un motivo de preocupación / Internet/. Washington DC: OMS; (citado 11 Ago 2014). Disponible en: http:// new.paho.org/hq/dmdocuments/2009/ AdolescentAnemiaSpan $\% 20 \% 282 \% 29$.pdf

5. Kurniawan YA, Muslimatun S, Achadi EL, Sastroamidjojo S. Anemia and iron deficiency anemia among young adolescent girls from the peri urban coastal area of Indonesia. Asia Pac J Clin Nutr. 2006; 15(3):350-6.

6. Nead KG, Halterman JS, Kaczorowski JM, Auinger $\mathrm{P}$, Weitzman $\mathrm{M}$. Overweight children and adoles-cents: A risk group for iron geficiency. Pediatrics. 2004; 114(1):104-8.

7. Doig K. Trastornos del metabolismo del hierro. En: Rodak BF, editor. Hematología. fundamentos y aplicaciones clínicas. 2da. ed. Buenos Aires: Editorial Médica Panamericana; 2010. p. 213-29.

8. Sanabria MC. Perfiles nutricionales por países: Paraguay /Internet/. Roma: Organización de la Naciones Unidad para la Agricultura y la Alimentación; 2001. (citado 10 Sep 2014). Disponible en: ftp://ftp.fao.org/ag/agn/ nutrition/ncp/pry.pdf

9. Instituto Nacional de Alimentación y Nutrición. Programa alimentario nutricional integral: Resumen [Internet]. Asunción: Instituto Nacional de Alimentación y Nutrición. [citado 12 Dic 2014]. Disponible en: h t t p : / / ww w.inan.gov.py/docs / resumen_ejecutivo_pani.pdf

10. Decreto 20830: Por el cual se declara obligatorio el enriquecimiento de la harina de trigo con hierro y vitaminas /Internet/. Asunción. (28 abril 1998). Disponible en: http://www.inan.gov.py/docs/Decreto\% 2020830\%201998.pdf

11.Dirección General de Estadísticas, Encuestas y Censos. Pte Hayes. En: Atlas censal del Paraguay 2002 /Internet/. Fernando de la Mora: DGEEC; 2001. p.199-205. (citado 11 Ago 2014). Disponible en: http:// www.dgeec.gov.py/Publicaciones/Biblioteca/ Atlas $\% 20$ Censal\%20del $\% 20$ Paraguay/ $18 \%$ 20Atlas\%20Pte. $\% 2$ 20Hayes\%20censo.pdf

12.Díaz RV, Sosa L, Guillén R, Pistilli N, Páez M, Almirón M, et al. Prevalencia de anemia en varias comunidades de la etnia Nivaclé del Chaco paraguayo. Mem. Inst. Investig. Cienc. Salud. 2013; 11(1): 15-21.

13. Echague G, Sosa L, Díaz V, Funes P, Ruíz I, Pistilli $\mathrm{N}$, et al. Anemia en niños indígenas y no indígenas menores de 5 años de comunidades rurales del Departamento de Caazapá. Pediatr. (Asunción). 2013; 40(1):19-28.

14. World Health Organization. Reproductive health indicators: Guidelines for their generation, interpretation and analysis for global monitoring /Internet/. Geneva: WHO; 2006. [citado 29 Ago 2014]. Disponible en: h t t p : / / w h q libdoc. who.int / publications/2006/924156315X_eng.pdf

15.Redondo Martínez JV, Pedotti Vázquez R, Serafin P. Guías alimentarias del Paraguay / Internet/. Asunción: Instituto Nacional de Alimentación y Nutrición; 2011. [citado 1 Oct 2014]. Disponible en: http:// inan.gov.py/oldweb/matdidact.html

16.Canela R, Schapt N, Redondo J, Bécker D, 
Riveros F, Gómez MA, et al. Guías alimentarias del Paraguay /Internet/. 2a act. Asunción: Instituto Nacional de Alimentación y nutrición; 2015. [citado 1 Oct 2014]. Disponible en: http://inan.gov.py/newweb/? page_id $=455$

17.Instituto Nacional de Alimentación y Nutrición (INAN). Alimentación para el escolar con desnutrición /Internet/. Asunción: INAN. [citado 1 Oct 2014]. Disponible en: http:// w w w . inan.gov.py/oldweb/graficos / cartillaescolardesnut.pdf

18. World Health Organization. Global Database on Body Mass Index /Internet/. WHO; 2006. BMI classification [citado 1 Oct 2014]. http:// a p p s. w ho. in t/b m i / i ndex.js p ? introPage=intro_3.html

19. Campuzano Maya G. La clínica y el laboratorio: Del hemograma manual al hemograma de cuarta generación. Medicina \& Laboratorio. 2007; 13(11-12):511-50.

20.Khusun $H$, Yip R, Schultink W, Dillon DH. World Health Organization hemoglobin cut-off points for the detection of anemia are valid for Indonesian population. J Nutr. 1999; 129 (9):1669-74.

21. Casanueva E, Regil LM, Flores-Campuzano MF. Anemia por deficiencia de hierro en mujeres mexicanas en edad reproductiva: historia de un problema no resuelto. Salud pública Méx. 2006; 48(2):166-75.

22. Ríos Castillo I, Brito A, Olivares M, Lopez de Romaña D, Pizarro F. Low prevalence of iron deficiency anemia between 1981 and 2010 in Chilean women of childbearing age. Salud pública Mex. 2013; 55(5):478-83.

23. Echagüe G, Díaz V, Pistilli N, Méndez J, Ríos $R$, Nuñez D, et al. Valores hematológicos en donantes de bancos de sangre de Asunción, Paraguay. Mem. Inst. Investig. Cienc. Salud. 2003; 2(1): 49-56.

24. Manjarrés LM, Díaz A, Carriquiry A. Asociación entre la ingesta de nutrientes hematopoyéti$\cos$ y el origen nutricional de la anemia en mujeres en edad fértil en Colombia. Rev Panam Salud Pública. 2012; 31(1): 68-73.

25. Organización Mundial de la Salud. Enfermedades no transmisibles y salud mental: Enfermedades no transmisibles. Perfiles de países 2014 /Internet/. OMS; 2014. [citado 5 Mar 2015]. Disponible en: http://www.who.int/ $\mathrm{nmh} /$ countries/es/

26. Barría P RM, Amigo C H. Transición nutricional: Una revisión del perfil latinoamericano. ALAN. 2006; 56(1): 3-11.
27.Thompson B. Food-based approaches for combating iron deficiency. Nutritional anemia /Internet/. Rome: Food and Agriculture Organization (FAO). [citado 2 Mar 2015]. Disponible en: ftp://ftp.fao.org/ag/agn/ nutrition/Kapitel_21_210207.pdf

28.Pedraza DF. Obesidad y pobreza: marco conceptual para su análisis en Latinoamérica. Saude soc. $2009 ; 18(1): 103-17$.

29.Baudrand R, Arteaga E, Moreno M. El tejido graso como modulador endocrino: Cambios hormonales asociados a la obesidad. Rev. méd. Chile. 2010; 138(10):1294-301.

30.De la Calle M, Armijo O, Martín E, Sancha M, Magdaleno F, Omeñaca F, et al. Sobrepeso y obesidad pregestacional como factor de riesgo de cesárea y complicaciones perinatales. Rev. chil. obstet. ginecol. 2009; 74 (4):233-8.

31.Dirección General de Estadísticas, Encuestas y Censos. Paraguay: Mapas de pobreza por departamentos y distritos: Departamento de Presidente Hayes. En: Indicadores básicos para focalizar el gasto social en $\mathrm{Pa}$ raguay /Internet/. Fernando de la Mora: DGEEC; [citado 11 Agosto 2014]. Disponible en: http: / / www.dgeec.gov.py/ Publicaciones/Biblioteca/IBF/IBF.htm

32. Ministerio de Salud Pública y Bienestar Social. Dirección General de Planificación. Dependencias. Análisis de situación de salud Eje Chaco: Presidente Hayes, Boqueròn, Alto Paraguay /Internet/. Asunciòn: Ministerio de Salud Pùblica y Bienestar Social; 2012. [citado 11 Agosto 2014]. Disponible en: http://www.mspbs.gov.py/planificacion/ wp-content/uploads/2013/01/EJE-5CHACO.pdf

33.Dirección General de Estadística, Encuestas y Censos. Principales resultados de pobreza y distribución del ingreso /Internet/. Fernando de la Mora: DGEEC; 2013. [citado Mar 2 2015]. Disponible en: http:// www.dgeec.gov.py/Publicaciones/Biblioteca/ eph2014/Boletin \%20 de\%20 pobreza\% 202014.pdf

34.Dirección General de Estadística, Encuestas y Censos. Atlas de necesidades básicas insatisfechas 2005 /Internet/. Fernando de la Mora; DGEEC; 2005. [citado 2 Mar 2015]. Disponible en: http://www.dgeec.gov.py/ Publicaciones/Biblioteca/Atlas\%20NBI/0a\% 20NBI\%20Presentacion.pdf 